\title{
PAPER
}

\section{Selective and faithful imitation at 12 and 15 months}

\section{Elma E. Hilbrink, ${ }^{1,2}$ Elena Sakkalou, ${ }^{1,3}$ Kate Ellis-Davies, ${ }^{1,4}$ Nia C. Fowler ${ }^{1}$ and Merideth Gattis ${ }^{1}$}

1. School of Psychology, University of Cardiff, UK

2. Max Planck Institute for Psycholinguistics, Nijmegen, The Netherlands

3. Neurosciences Unit, UCL Institute of Child Health, UK

4. School of Psychology, University of Cambridge, UK

\begin{abstract}
Research on imitation in infancy has primarily focused on what and when infants imitate. More recently, however, the question why infants imitate has received renewed attention, partly motivated by the finding that infants sometimes selectively imitate the actions of others and sometimes faithfully imitate, or overimitate, the actions of others. The present study evaluates the hypothesis that this varying imitative behavior is related to infants' social traits. To do so, we assessed faithful and selective imitation longitudinally at 12 and 15 months, and extraversion at 15 months. At both ages, selective imitation was dependent on the causal structure of the act. From 12 to 15 months, selective imitation decreased while faithful imitation increased. Furthermore, infants high in extraversion were more faithful imitators than infants low in extraversion. These results demonstrate that the onset of faithful imitation is earlier than previously thought, but later than the onset of selective imitation. The observed relation between extraversion and faithful imitation supports the hypothesis that faithful imitation is driven by the social motivations of the infant. We call this relation the King Louie Effect: like the orangutan King Louie in The Jungle Book, infants imitate faithfully due to a growing interest in the interpersonal nature of interactions.

A video abstract of this article can be viewed at http://youtu.belqlnIoflk-u8.
\end{abstract}

\section{Introduction}

Much of the research on imitation has been concerned with what and when infants and young children are able to imitate. The results of this research indicate that infants are capable of copying facial gestures from birth, vowel sounds from around 4 months, and simple actions on objects between 6 and 12 months (Barr, Dowden \& Hayne, 1996; Kuhl \& Meltzoff, 1996; Meltzoff \& Moore, 1983). By comparison few studies have addressed why infants imitate. In this paper, we report a longitudinal study investigating the relation between developmental changes in why infants imitate and what infants imitate.

In a seminal paper, Uzgiris (1981) suggested two motivations to imitate: an instrumental motivation to learn new skills, and a social motivation to create and share experience with social partners. In both cases imitation is dependent on the cognitive level of the imitator but for different reasons. In the case of instrumental motivation, cognitive level influences the imitator's understanding of actions. In the case of social motivation, cognitive level influences the imitator's understanding of interactions, in particular their interpersonal nature. Uzgiris suggested that as cognitive level changes, so does the motivation to imitate. Initially infants imitate for instrumental reasons, which Uzgiris described as resolving a puzzle. During the second year of life, they begin to imitate for social reasons, which Uzgiris described as maintaining a game.

Importantly, the evidence on which Uzgiris based her proposal differed for the two motivations. To illustrate the instrumental motivation to imitate, she reviewed experimental evidence, and to illustrate the social motivation to imitate, she primarily reviewed observational evidence. In her review, experimental evidence of the social motivation to imitate was limited to one study illustrating that the child's understanding of interpersonal situations changes around 16 months. In that 
study, Killen and Uzgiris (1981) tested four age groups $\left(7 \frac{1}{2}, 10,16\right.$ and 22 months) on imitation of three types of action on objects: very simple actions (e.g. shaking a doll), socially appropriate actions (e.g. bringing a cup to the mouth), and socially inappropriate actions (e.g. bringing a car to the mouth). Killen and Uzgiris (1981) reasoned that if infants copy for purely instrumental reasons, they would observe an age-related shift away from copying simple actions. This was not the case. The youngest group copied simple actions only; the middle age groups (10- and 16-month-olds) copied simple actions and socially appropriate actions; and the eldest group copied all three types of action, including socially inappropriate actions. Uzgiris (1981) concluded that by 22 months, infants understand imitation as a social exchange and are motivated to maintain social interactions by imitating the actions of a social partner, including simple actions they already fully understand.

Over the past two decades, several studies have demonstrated that imitation varies not only according to age and cognitive level, but also according to context. On some tasks, children imitate selectively, reproducing a subset of the actions of others. For example, Carpenter, Akhtar and Tomasello (1998) showed 14- to 18-montholds two-action sequences on a novel toy. One of the actions was marked accidentally (Whoops!), and one of the actions was marked intentionally (There!). Most infants imitated the demonstrations selectively, and reproduced the intentionally marked action (see also Sakkalou \& Gattis, 2012 for a recent extension on this study). On other tasks, however, children imitate faithfully, even to the extent of copying unnecessary steps in an action sequence, or overimitating. For example, Horner and Whiten (2005) showed 3- to 4-year-olds a sequence of actions to open a box and retrieve a sticker. Some of the actions were causally necessary, and other actions were causally unnecessary. Some of the demonstrations involved a clear box that made it possible for children to observe the causal relations and thus distinguish the necessary and unnecessary actions, while other demonstrations involved an opaque box that prevented children from distinguishing necessary actions from unnecessary actions. Children copied both necessary and unnecessary actions, demonstrating faithful imitation even when they could see that some steps were causally unnecessary.

The dominant explanation for selective imitation is that infants and children are sensitive to the goaldirected nature of human actions and selectively reproduce the goals of others (Bekkering, Wohlschläger \& Gattis, 2000; Gattis, Bekkering \& Wohlschläger, 2002). The results of several studies support the claim that infants and children copy goals and intentions and omit mistakes and other aspects of observed behavior (Bekkering et al., 2000; Carpenter et al., 1998; Carpenter, Call \& Tomasello, 2005; Gardiner, Greif \& Bjorklund, 2011; Gattis et al., 2002; Hoicka \& Gattis, 2008; Over \& Gattis, 2010; Perra \& Gattis, 2008; Sakkalou, EllisDavies, Fowler, Hilbrink \& Gattis, 2013).

Such selectivity also draws on causal understanding, however, as demonstrated by Brugger, Lariviere, Mumme and Bushnell (2007). To investigate whether infants use causal knowledge to selectively imitate, Brugger et al. (2007, Study 1) showed 14- to 16-month-olds a two-action sequence in two different causal contexts. In the necessary condition the first action was causally necessary in order to prepare for the second action. In the unnecessary condition the first action was unnecessary. Infants copied the first action more often when it was causally necessary than when it was not, demonstrating that selective imitation depends on understanding of the causal structure embedded in behavior, as well as a sensitivity to goals and intentions.

Two explanations for faithful imitation have been proposed. Drawing on Uzgiris' distinction between instrumental and social motivations to imitate, Nielsen (2006) proposed that from 18 months onward, children become less concerned with the logical structure of action, and more concerned with social experience, leading to an increase in faithful imitation. To test this explanation, he compared children's tendency to copy versus simplify an experimenter's method for opening a box under two conditions (2006, Study 3). In the social condition the experimenter interacted with children throughout the study, including normal eye contact and social responsivity. In the aloof condition the experimenter avoided eye contact and social interaction with the child. In both conditions the experimenter opened a series of boxes by using a tool to move a switch that could be more easily moved with the hand. Eighteen-month-olds were more likely to use the tool in the social condition than in the aloof condition. Twenty-four-month-olds were consistent in their tool use in both conditions but were more successful in opening the box when the model acted socially. In contrast, in a related experiment using a normal social condition, Nielsen showed that 12-month-olds generally opened the box with their hands, and used the tool to open the box only when they had seen a failed hand demonstration followed by a successful tool demonstration. Nielsen concluded that social context and age both influence social motivations to imitate, with children becoming more faithful imitators around 18 to 24 months.

Nielsen's proposal is further supported by the results of a second study from Brugger et al. (2007). Like Nielsen, Brugger et al. manipulated the social context by 
varying the model's behavior: in the socially cued condition the first action was accompanied by clear ostensive cues directing the infant's attention to the action, and in the not cued condition, the first action was preceded instead by the model looking at a wall and commenting on the weather. Infants copied the first action more frequently in the socially cued condition, or in other words, when the model acted in a natural social manner.

In contrast to the social accounts of faithful imitation proposed by Nielsen (2006) and Brugger et al. (2007), others have argued that faithful imitation arises as a byproduct of causal learning processes (Lyons, Damrosch, Lin, Macris \& Keil, 2011; Lyons, Young \& Keil, 2007). Lyons and colleagues (2007) proposed that children automatically encode adults' actions as causally informative. In their view, faithful imitation is one consequence of this automatic causal encoding, particularly in unfamiliar situations. Thus, according to their account, faithful imitation occurs in the absence of causal understanding.

Based on the evidence reviewed above, we investigated whether correspondences exist between selective imitation and instrumental motivations to imitate, and between faithful imitation and social motivations to imitate. This builds on and goes beyond Uzgiris (1981) because it proposes specific links between why children imitate and what they imitate. Over and Carpenter (2012) recently reviewed research on imitation in children and adults and proposed that any account that wishes to explain both selective and faithful imitation needs to take into account the social nature of imitation. More specifically, they proposed that young children's variable imitative behavior might be explained by the different goals children might hold, such as learning goals that can lead to selective imitation and social goals that can lead to faithful imitation (Carpenter, 2006; Over \& Carpenter, 2012). This proposal is similar to what we set out to assess in the present paper. Our goal was to assess a possible correspondence between selective imitation and instrumental motivations and between faithful imitation and social motivations. Therefore we conducted a longitudinal study using a single experimental paradigm to investigate developmental changes in selective and faithful imitation. We wanted to identify the earliest onset of faithful imitation, and to capitalize on individual differences in social motivation to investigate whether faithful imitation is related to the motivation to maintain social interactions.

We examined the proposed correspondence between selective imitation and instrumental motivations and between faithful imitation and social motivations longitudinally at 12 and 15 months using an experimental paradigm developed by Brugger et al. (2007), and described above. The study involved a within-subjects design, where an experimenter modeled a two-action sequence on a novel toy in two different causal contexts: one context where the first action was causally necessary, enabling the second action, and one context in which the first action was causally unnecessary, or irrelevant to the second action. Similar to Brugger et al.'s second experiment we focused in our analyses on imitation of the first demonstrated actions only. The reason we focused on the first actions was that this is what differed between the two conditions: half of the time the first action was relevant (necessary) to the second action and half of the time irrelevant (unnecessary) to the second action. In contrast, the second actions remained constant between the conditions. We expected that at 12 months infants would produce more selective imitation as evidenced by imitating more causally relevant actions. We therefore operationalized selective imitation as copying more first actions in the necessary condition than first actions in the unnecessary condition. On the other hand, faithful imitation involves copying in a more exact manner (Over $\&$ Carpenter, 2012). We therefore defined faithful imitation as copying irrelevant first actions in the unnecessary conditions, while also copying necessary first actions in the necessary conditions. We expected that this pattern of responding would occur more with increasing age. We quantified faithful imitation as the total number of first actions copied in both unnecessary and necessary conditions. However, it is possible that the first actions in the necessary condition, by virtue of being necessary, are nearly always copied and therefore are relatively stable over time. It is therefore likely that age effects may show in the unnecessary first actions. The longitudinal design was critical to our aims: testing infants in the same experimental paradigm at 12 and 15 months allowed us to assess whether infants demonstrated causal understanding of the task, as indexed by selective imitation. Furthermore, after having demonstrated causal understanding, we could then assess whether those same infants subsequently shifted from selective to faithful imitation.

We adopted an infant-focused individual differences strategy for evaluating the influence of social motivations on imitation. One problem with interpreting the results of Brugger et al. (2007) and Nielsen (2006) is that when a model is acting aloof, infants will almost certainly decrease in performance on any task, thus weakening the logical inference that social motivations lead to increased imitation. To avoid this problem, we evaluated infants' social motivations by assessing extraversion. Extraversion, of which surgency is the infant antecedent, is a critical factor in all major systems for 
describing and measuring personality and temperament, collectively called 'the Big Five' (Buss \& Plomin, 1986; Gartstein \& Rothbart, 2003; Goldsmith, Buss, Plomin, Rothbart, Thomas, Chess, Hinde \& McCall, 1987; Putnam, Gartstein \& Rothbart, 2006; Rothbart, Ahadi, Hershey \& Fisher, 2001). In a recent study, Grist and McCord (2010) compared temperament, including surgency, as measured by the Children's Behavior Questionnaire (Rothbart et al., 2001) with a measure they specifically designed to assess the Big Five Personality traits. Their findings demonstrated a strong significant relation between surgency and extraversion. Furthermore, although various measures of temperament differ in the specific scales contributing to extraversion/surgency, the global factor structure is consistent across measures: extraversion/surgency indexes an individual's desire to be with other people, to prefer shared activities, to initiate social contact, and to be socially responsive (Goldsmith et al., 1987). We reasoned that if an emerging awareness of interpersonal relations during the second year leads infants to imitate more faithfully, as suggested by Uzgiris (1981) and Nielsen (2006), infants high in extraversion would reach that shift slightly earlier compared to other infants, and thus be more likely to demonstrate faithful imitation at 15 months.

We assessed extraversion using the Early Childhood Behaviour Questionnaire (ECBQ), a parent report measure developed by Putnam et al. (2006). The ECBQ is a widely used measure of toddler temperament and together with the Infant Behavior Questionnaire-Revised (Gartstein \& Rothbart, 2003) and the Children's Behaviour Questionnaire (Rothbart et al., 2001) it allows assessment of the major components of temperament during infancy from 3 months up to 7 years of age. Validation of the ECBQ has revealed the factor surgency as the childhood antecedent of extraversion. In the $\mathrm{ECBQ}$, the scales loading onto extraversion/surgency are impulsivity, activity level, high-intensity pleasure, sociability, and positive anticipation. Importantly, longitudinal studies have demonstrated stability of extraversion/ surgency across development, even across different measures with different contributing scales, such as those that do or do not include sociability (Calkins, Fox \& Marshall, 1996; Putnam, Rothbart \& Gartstein, 2008). In addition, when Putnam et al. (2008) assessed longitudinal continuity of temperament throughout infancy and early childhood using the IBQ-R, the ECBQ and the $\mathrm{CBQ}$, their analyses revealed that extraversion/ surgency was stable across all time points with moderate to large correlations. However, Putnam and colleagues (2006) suggest that caution is necessary when using some of the ECBQ scales separately, especially with regard to the scale impulsivity as it performed relatively poorly on internal consistency measures. Furthermore, they note that due to the large number of scales included in the ECBQ there are relatively few items measuring each dimension. For these reasons, we used the overall factor scores for extraversion/surgency to assess the social motivation to imitate, rather than the component scales.

By combining an experimental measure of selective and faithful imitation with a parent report measure of temperament in a longitudinal design, we were able to address three aims. Our first aim was to evaluate infants' causal understanding and the proposed correspondence between selective imitation and instrumental motivation. Building on Brugger et al. (2007), we hypothesized that selective imitation based on infants' causal understanding could be observed longitudinally at both 12 and 15 months. Our second aim was to identify selective and faithful imitation on the same experimental task. We hypothesized that from 12 to 15 months, faithful imitation would increase. To accurately evaluate this hypothesis, it was essential to measure both selective and faithful imitation on the same task and among the same infants. Our third aim was to evaluate the proposed correspondence between faithful imitation and social motivation. By identifying the earliest onset of faithful imitation and at the same time assessing individual differences in social motivation, we sought to evaluate whether the desire to share experience with social partners leads infants to begin to imitate more faithfully in their second year.

\section{Method}

\section{Participants}

Thirty-seven healthy infants were tested at 12 months ( $M=365$ days, $\quad$ range $=354$ to 371 days $)$ and 15 months $(M=458$, range $=446$ to 465 days $)$ as part of the First Steps longitudinal study. Expecting parents were recruited into First Steps during pregnancy through news announcements and from local organizations and events. Full demographics on this sample are available in Ellis-Davies, Sakkalou, Fowler, Hilbrink and Gattis (2012). The First Steps design involved monthly testing of infants on various aspects of sociocognitive development from 2 until 18 months. Three types of data were collected at the monthly testing sessions: experimental, observational, and parent report. Parent report measures included temperament measures at 4 and 15 months, and vocabulary measures from 12 months onward. In addition to the monthly sessions, parents kept electronic diary records of behaviors occurring in the home environment from birth to 18 months. At each monthly 
testing session, families were given shopping vouchers and a small gift in return for their participation.

\section{Apparatus}

Stimuli were adapted from Brugger and colleagues (2007). Four toys were used: two wooden boxes containing a hidden toy, and two toy trucks, each containing two toy animals, one in the front seat and one in the back seat. Pushing the animal in the front seat of the truck caused music to play and the wheels to spin (see Figure 1). The truck was presented in its commercial packaging to keep it stationary. Placing the truck out of its packaging would cause it to 'drive' across the table upon pushing the animal in the front. Each toy type was used once in the necessary condition and once in the unnecessary condition, with modifications for each condition so that there were four different toys in total. For the box in the necessary condition a Velcro strap was attached to the lid of the box that needed to be removed before the lid could be opened. For the box in the unnecessary condition the Velcro strap was attached to the other half of the box, next to the lid. Therefore it was unnecessary to remove the strap before opening the lid. For the truck in the necessary condition a square cover made of plexi-glass with a knob attached to the top was placed over the animal in the front seat of the truck. Therefore the cover needed to be removed in order to push the animal. In the unnecessary condition the cover was placed over the animal in the back of the truck and it was therefore unnecessary to remove the cover before pushing the animal in the front seat. To minimize
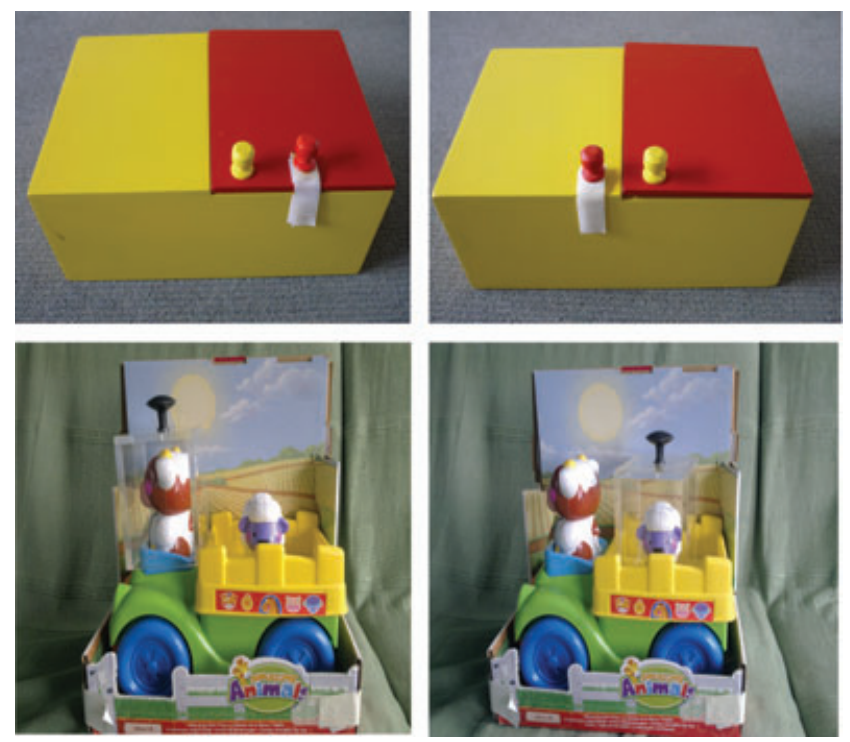

Figure 1 The toys used in the necessary condition (left) and the unnecessary condition (right). memory effects, a new set of toys was used at 15 months. The new set was composed of four toys similar to those described above, but painted in different colors.

\section{Procedure}

Infants faced a table, and were seated on the lap of a secondary experimenter or on their mothers' lap. Mothers were instructed not to speak to their infants or help their infants in any way. The primary experimenter sat at the same table, $90^{\circ}$ to the infant's right. As soon as the infant was settled the experimenter brought out the first toy from under the table. A two-step sequence was modeled twice followed by a response period. Each modeling period started with the experimenter saying, while looking at the infant: 'Look what I've got! Shall I show you how it works?', followed by the two-step sequence. For the box toy, regardless of condition, the sequence consisted of removing the Velcro strap and opening the lid to reveal the hidden toy. The only difference between conditions was the position of the Velcro strap: either on the lid (necessary) or next to the lid (unnecessary). After the first demonstration the experimenter said, 'Shall I show you again?', and repeated the demonstration. After the second demonstration, the experimenter said, 'Would you like to play with the toy?', and moved the toy within reach of the infant. The response period began when the infant first touched the toy and ended when the infant had found the hidden toy inside or when a maximum of 60 seconds had passed without the infant succeeding. After the response period the toy was taken away and put out of view of the infant and the next toy was brought out on the table. For the truck, regardless of condition, the sequence consisted of removing the plastic cover and pushing the animal on the front to make the music play and the wheels spin. The only difference between conditions was whether the cover was placed over the animal in the front (necessary condition) or over the animal in the back (unnecessary condition). Infants were shown both types of toys in both conditions in one of four possible orders counterbalanced across infants: (1) box necessary, truck unnecessary, box unnecessary, truck necessary, (2) box unnecessary, truck necessary, box necessary, truck unnecessary, (3) truck necessary, box unnecessary, truck unnecessary, box necessary, (4) truck unnecessary, box necessary, truck necessary, box unnecessary.

To measure extraversion, mothers completed the Early Childhood Behavior Questionnaire (ECBQ) at 15 months. Following Putnam et al. (2006), the factor surgency consisted of a mean score based on five scales: activity level, high-intensity pleasure, impulsivity, positive anticipation, and sociability. Surgency is the only 
factor score from the ECBQ reported here. Mothers also completed the Infant Behavior Questionnaire-Revised (IBQ-R) (Gartstein \& Rothbart, 2003) at 4 months. The IBQ- $R$ is not reported here, but we refer to it in the preliminary analyses below that assessed stability of extraversion/surgency over time.

\section{Coding and analyses}

Each testing session was recorded and later coded from video by a trained coder using the Mangold Interact software for coding behavioral data. The coder was blind to the hypotheses and the surgency scores, but not the condition because the toys were visible in the video. The coder coded infant actions after receiving the toy until the end of the 60-second response period. Our primary focus was on the first action and whether it matched the first action of the experimenter, removing the Velcro strap for the boxes or removing the cover for the trucks. A second coder scored $25 \%$ of the videos to allow assessment of reliability (Kappa $=0.94, p<.05$ at 12 months and Kappa $=1, p<.05$ at 15 months).

Selective imitation was defined as copying the first action more often in the necessary condition than the unnecessary condition. Faithful imitation was defined as copying both necessary and unnecessary first actions. However, as mentioned earlier it is possible that the number of first actions copied in the necessary condition, by virtue of being necessary, is relatively stable over time. It is therefore likely that age effects may show in the unnecessary first actions

\section{Results}

To analyze the data we first conducted two preliminary analyses on the surgency data. The first analysis concerned the stability of the surgency measure from the first few months of life until 15 months. The second analysis was performed to split the infants into a highsurgency group and a low-surgency group. Next, we conducted our main analyses using mixed logit modeling in $\mathrm{R}$ ( $\mathrm{R}$ Development Core Team, 2012), using the LME4 package (Bates, Maechler \& Dai, 2012). A recent paper by Jaeger (2008) has demonstrated that it is more appropriate to use mixed logit modeling to analyse repeated measures categorical data, rather than ANOVAs (even after transforming the data). He demonstrated that using ANOVAs on this type of data led to spurious results because of over-fitting. Another advantage of using mixed logit models is that mixed logit models have a greater power and it is therefore more likely that mixed logit models detect true effects. Furthermore, a recent study by Hoicka and Akhtar (2011) demonstrated that when comparing the results of the mixed logit analyses on their data with the results of ANOVAs, they were highly similar. However, the mixed logit models were also able to capture additional effects. For these reasons we have analysed our data using mixed logit modeling in R.

\section{Preliminary analyses}

To assess whether surgency was a stable factor in our sample, longitudinal analyses were conducted comparing factor scores on surgency from the IBQ-R at 4 months and the ECBQ at 15 months. These analyses demonstrated stability of extraversion/surgency from 4 to 15 months, $r=.43 p<.05$. This finding supported our decision to use the factor surgency as measured at 15 months in the present study to assess the relation between social motivations to imitate and faithful imitation at 12 and 15 months. Infants were divided into a high-surgency group and a low-surgency group using a median split.

\section{Main analyses}

For the main analyses we followed the procedure used by Hoicka and Akhtar (2011). First a base model was built which included an intercept and ID number and type of toy as random variables and first actions copied (yes, no) as dependent variable. The base model was then compared to models including Condition (necessary, unnecessary), Age (12 months, 15 months), Surgency (low, high), Order (each of the four possible orders) and Gender (male, female). If one of these models was a significant improvement compared to the base model the variable was added to the model. If more than one variable improved the model, the variable with the lowest log-likelihood was added to the base model and the remaining variables were checked again for whether they improved the model or not. Furthermore, we checked for any interaction effects (for a more detailed description of the procedure see Hoicka \& Akhtar, 2011). All effects are expressed in odds ratios; when the odds ratio of an event is greater than one, the event is more likely to happen than not, and when the odds are less than one it is more likely the event will not happen.

The base model was improved by adding Condition (necessary, unnecessary), $\chi^{2}(1)=34.38, p<.001$, Surgency (low, high), $\chi^{2}(1)=4.23, p<.05$, and an interaction of Condition (necessary, unnecessary) and Age (12 months, 15 months), $\chi^{2}(2)=7.83, p<.05$. There were no Gender or Order effects. As can be seen in Figure 2, in the necessary condition the first actions of the two-step sequence are nearly always copied (at 


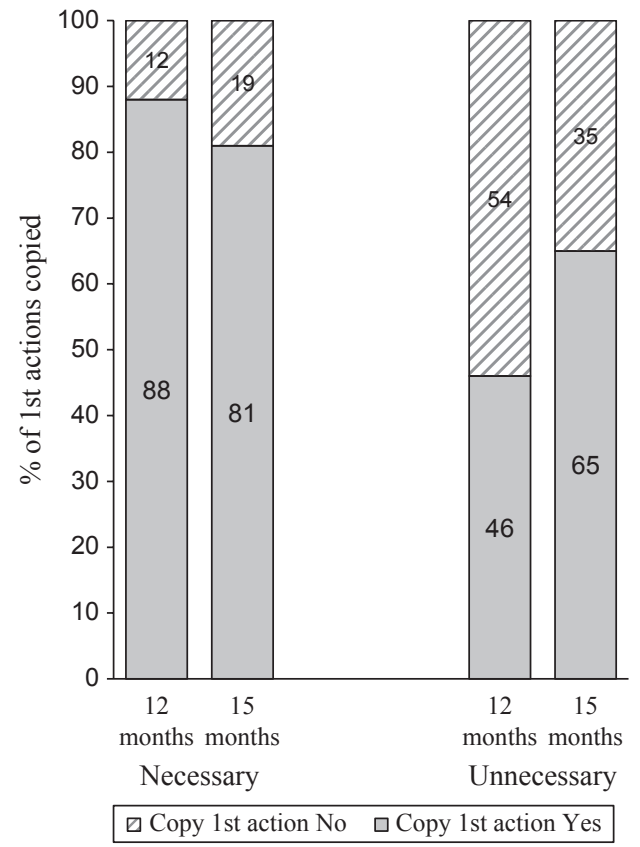

Figure 2 The percentage of actions copied at each age in each condition.

12 months $88 \%$ of the first actions in the necessary condition are copied) and also remain fairly constant over time ( $81 \%$ at 15 months), while in the unnecessary condition this is clearly less so $(46 \%$ at 12 months and $65 \%$ at 15 months). Figure 3 displays the percentage of first actions copied by high- and low-surgency infants (divided by a median split), demonstrating that highsurgency infants copy more first actions (76\% of first actions are copied) compared to low-surgency infants $(63 \%)$.

The resulting model (loglikelihood $=-154.38$, $N=296$ ) included significant effects of Condition

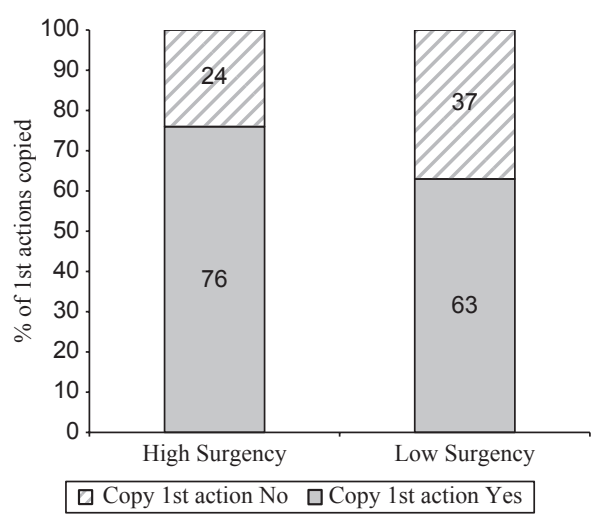

Figure 3 The percentage of 1st actions copied by high-and low-surgency infants collapsed across age. (unnecessary vs. necessary), $O R=0.0003, p<.05$, Surgency (low, high), $O R=0.45, p<.05$, and an interaction between Condition and Age, $O R=1.65, p<.05$. The small odds ratio (0.0003) for Condition indicates that it is highly unlikely for infants to copy first actions in the unnecessary condition compared to the necessary condition. In other words, the effect of Condition shows that infants are far more likely to copy first actions in the necessary condition compared to the unnecessary condition. The Condition by Age effect demonstrates that infants are 1.6 times more likely to copy first actions in the unnecessary condition at 15 months compared to at 12 months. The odds ratio of 0.45 for Surgency indicates that the low-surgency group is less likely to copy first actions compared to the high-surgency infants.

To further explore the Age by Condition effect we ran the same analyses again but separately for 12 months and 15 months. At 12 months the base model was improved by adding Condition (unnecessary, necessary), $\chi^{2}(1)=32.82, p<.00$. The resulting model at 12 months (loglikelihood $=-76.33, N=148)$ therefore included a significant effect of Condition (unnecessary, necessary), $O R=0.09, p<.00$. The low odds ratio indicates that at 12 months it is unlikely that infants will copy first actions in the unnecessary condition compared to the necessary condition. At 15 months the base model was also improved by including Condition (unnecessary, necessary), $\chi^{2}(1)=6.62, p=.01$, and the resulting model (loglikelihood $=-75.98, N=148)$ therefore included a significant effect of Condition (unnecessary, necessary), $O R=0.22, p=.01$. Thus at 15 months, similar to the findings at 12 months, infants are less likely to copy first actions in the unnecessary condition compared to the necessary condition. However, the odds ratio at 15 months is not as small as at 12 months, indicating that it is more likely that infants will copy first actions in the necessary condition at 15 months than at 12 months.

To further explore this finding we assessed the effect of Age (12 months, 15 months) when running the analyses separately for the necessary condition and the unnecessary condition. For the unnecessary condition the base model was improved by adding Age (12 months, 15 months), $\chi^{2}(1)=6.05, p=.01$. The resulting model had a loglikelihood $=-97.81, N=148$ with a significant effect of Age (12 months, 15 months), $O R=1.34$, $p=.01$. Thus, at 15 months infants were 1.3 times more likely to copy first actions in the unnecessary condition compared to at 12 months. For the necessary condition no effect of Age was found.

The effect of Surgency indicated that high-surgency infants are more likely to copy first actions than lowsurgency infants. To address the possible explanation that high-surgency infants are simply more active and 
therefore copy more actions in general, we assessed whether, in addition to copying more first actions, highsurgency infants also copied more second actions. We ran the same mixed logit analyses but instead of having First actions copied as the dependent variable we ran the analyses with Second actions as dependent variable. Adding Surgency to the base model did not improve the model (nor did any of the other variables), $\chi^{2}(1)=.89$, $p>.05$. This means high- and low-surgency infants were equally likely to copy the second actions. Furthermore, the number of second actions did not vary with increasing age, nor did it differ between the necessary and unnecessary conditions.

\section{Discussion}

We conducted a longitudinal study investigating the relation between developmental changes in why infants imitate and what infants imitate. In particular, we wanted to investigate developmental changes in selective and faithful imitation, and whether those changes are influenced by the motivation to maintain social interactions. Our aim was to compare selective and faithful imitation in a single experimental task, and to investigate the social motivation to imitate without the inherent confounds of designs using an aloof experimenter.

In our study, selective and faithful imitation were measured at 12 and 15 months using an imitation paradigm developed by Brugger et al. (2007), consisting of a two-action sequence in which the first action was either causally necessary or causally unnecessary for a second action. We defined selective imitation as copying more first actions in the necessary condition compared to the unnecessary condition. We defined faithful imitation as copying first actions in both the necessary and unnecessary conditions, i.e. the total number of first actions copied. We considered first actions only because the difference between the two conditions lies in the first actions which are either necessary or not, while the second actions do not differ between the two conditions. In addition, we reasoned that the second action was a form of outcome, as pulling on the lid opened the box, and pushing on the animal made music play. Similar to Horner and Whiten (2005), we were interested in whether the strategy used by the infants differed depending on the condition, not whether they can reproduce an end result. This definition of faithful imitation is also in line with that of the original study by Brugger et al. (2007), enabling us to compare our findings to those of the original study. Social motivation was measured in the present study as surgency on the ECBQ (Putnam et al., 2006). The design of the study allowed us to evaluate the role of infants' causal understanding in selective imitation, to identify the emergence of faithful imitation on the same task and with the same infants, and to evaluate a proposed correspondence between faithful imitation and social motivation.

Our first aim was to assess whether infants were able to imitate selectively based on their causal understanding of the task. Analyses revealed that infants imitate selectively depending on the causal structure of an action sequence. Regardless of age, infants were far more likely to copy the first action in the necessary condition compared to the unnecessary condition, thus demonstrating an understanding of the causal relations embedded in the task. This result replicates the findings of Brugger et al. (2007), who reported that 15-month-olds copied selectively depending on the causal properties of the objects, and demonstrates that even 12-month-olds imitate selectively based on their causal understanding of actions.

Next we sought to identify whether infants, on the same task, begin to imitate faithfully near the beginning of the second year. Analyses confirmed our prediction that with increasing age infants become more faithful imitators. While the percentage of first actions copied in the necessary condition remained fairly stable with increasing age, we did find a Condition by Age interaction effect. At 15 months infants were more likely to copy first actions in the unnecessary condition compared to at 12 months. Furthermore, in the follow-up assessment in which the analyses were run separately for 12 and 15 months, the odds ratio for copying first actions in the unnecessary versus the necessary condition increased over time from 0.09 to 0.22 . Thus by the time infants were 15 months they had become less unlikely to copy first actions in the unnecessary condition compared to the necessary condition. In other words, they increased in the likelihood to copy unnecessary actions.

By assessing selective and faithful imitation on the same task and testing the same infants longitudinally we were able to clearly identify the relation between the two types of imitation. Even though infants understand the causal relations in the tasks at 12 months, as evidenced by the high percentage of first actions copied in the necessary condition versus the much lower percentage in the unnecessary condition, by 15 months infants have started to copy more first actions in the unnecessary condition and are thus becoming more faithful imitators. This is consistent with earlier findings by Nielsen (2006) demonstrating that faithful and selective imitation vary with age. Whereas Nielsen reported an increase in faithful imitation from 18 months onwards, however, our results demonstrate that this developmental change 
begins earlier in life: we observed an increase in faithful imitation between 12 and 15 months.

To evaluate whether social motivations lead infants to begin to imitate more faithfully, we examined the influence of temperament on the faithfulness of imitation. According to previous research, infants become increasingly more aware of the interpersonal nature of interactions during the second year of life (Nielsen, 2006; Tomasello, Carpenter, Call, Behne \& Moll, 2005; Uzgiris, 1981). Furthermore, the temperament factor surgency, the infant antecedent of extraversion, assesses the individual's desire to be with and interact with other people (Goldsmith et al., 1987). We reasoned that if faithful imitation arises from the social motivations of infants generally, and their increasing awareness of the interpersonal nature of interactions specifically, then infants high in surgency would begin to imitate faithfully slightly earlier than their peers with lower levels of surgency. Indeed, an effect of surgency was found. The higher infants' scores on surgency the more likely they are to copy first actions. This suggests a role for surgency or, in other words, a role for a social motivation to interact with other people. However, from the current findings it is difficult to determine whether surgency is the driving force in the developmental shift towards more faithful imitation during the 12- to 15-month period. Future research with a larger sample size is necessary to establish the exact influence of surgency on faithful imitation. Nevertheless, it is clear from our findings that infants copy selectively based on the causal structure of the task, that infants become more faithful imitators between 12 and 15 months of age and that the motivation to interact with others does play a role in faithful imitation, with high-surgency infants copying more first actions than their low-surgency peers. We propose that like the highly-extraverted orangutan King Louie in the Walt Disney film The Jungle Book, who sang 'I wanna be like you, I wanna walk like you, talk like you too', high-surgency infants imitate more faithfully than their peers because they have a greater interest in the interpersonal nature of interactions.

Our findings confirm and extend other research investigating the influence of social motivation on faithful imitation (see for example Carpenter, 2006; Nielsen, 2009; Nielsen \& Blank, 2011; Nielsen, Simcock \& Jenkins, 2008; Over \& Carpenter, 2009). Our findings are also consistent with Over and Carpenter's (2012) account of infants' and young children's flexible imitative behavior. They suggested that one important factor determining what infants copy is whether infants have a learning goal, a social goal or a combination of the two. One of the social goals children might have to imitate is to communicate they are like their social partners. One way of demonstrating this is to do as their social partner does. To support their account, Over and Carpenter pointed to numerous studies demonstrating the social function of imitation. In the adult literature, for example, Chartrand and Bargh (1999) reported findings demonstrating that imitating someone's bodily behaviors increases the imitated person's liking of the imitator. Chartrand and Bargh further demonstrated that adults who have higher scores on an empathy scale (i.e. ability to take someone else's perspective) are also more likely to imitate their interaction partner's bodily actions, such as face rubbing or foot kicking.

Several studies involving young children have found evidence for a social function of imitation in early childhood. For example, Nielsen and colleagues (Nielsen et al., 2008) demonstrated that 24-month-olds were more likely to copy the exact actions of a live model who was socially responsive compared to a videotaped model who was not able to provide contingent feedback. In another study Nielsen and Blank (2011) showed that preschoolers are more likely to copy irrelevant actions if the person who stayed present during testing and handed the toy to them was the one who demonstrated the irrelevant actions even though they had seen another adult modeling the more efficient method. Together with the earlier mentioned study by Nielsen (2006) in which 18month-olds were more likely to copy the exact actions when the model acted socially compared to when she was aloof and studies such as those of Brugger et al. (2007), Killen and Uzgiris (1981) and Uzgiris (1981), this provides support for a social function of imitation in early childhood. Our findings demonstrate a relation between surgency and faithful imitation shortly after the first birthday, and provide further support for the social nature of faithful imitation.

Our findings show that faithful imitation is not only influenced by whether or not the experimenter is acting socially, but also by whether or not the child is motivated to interact socially with the experimenter. Surgency, as measured on the ECBQ, consists of five scales: activity level, high-intensity pleasure, impulsivity, positive anticipation, and sociability. Our analyses used the factor score rather than scale scores because extraversion is best understood as a combination of the desire to be with people and social responsivity. In addition, the factor surgency has greater stability and reliability than the component scales. Furthermore, the results of studies investigating relations between sociability and shyness suggest that the desire to be with people is distinct from extraversion (Cheek \& Buss, 1981; Schmidt \& Fox, 1994). Extraversion is not just about the preference to be with others, it also importantly involves the tendency to interact with others. Nevertheless, it can be an interesting 
question for future research to assess the role of the various components of surgency in infants' imitative behavior. However, as Putnam and colleagues (2006) have suggested, one needs to use the separate subscales with caution.

Want and Harris (2002) argued that clear operational definitions are needed to distinguish different imitative phenomena and make theoretical progress. The design of our study allowed us to address several potential problems with defining selective and faithful imitation. First, one could argue that selective imitation, or the omission of some of the modeled actions, may be due to difficulties with attention or memory - and indeed, working memory appears to play a role in selective imitation at least some of the time, as argued by Perra and Gattis (2008). In our study, however, the inclusion of the necessary condition with the same toys and same actions makes it clear that infants can attend to, remember, and do the first action, and thus makes infants' omission of it in the unnecessary condition more meaningful and likely to be predicated on their interpretation of it as causally irrelevant. Second, one could argue that children copy faithfully due to a lack of understanding of the causal structure of a task (i.e. Lyons et al., 2007). Testing the same infants on the same paradigm longitudinally at 12 and 15 months allowed us to assess their causal understanding of the task and to ask whether faithful imitation occurs in the presence or absence of causal understanding. Analyses regarding the Condition by Age interaction effect confirmed our hypothesis that at 12 months infants already demonstrate understanding of the causal structure of the task by copying necessary actions selectively. Importantly, despite demonstrating causal understanding at 12 months, infants subsequently increase in faithful imitation at 15 months, as evidenced by the increase in the likelihood of copying first actions in the unnecessary condition by the time infants are 15 months. Because the task involves very similar objects and actions at both ages, older infants can be expected to have at least the same understanding of the causal relations as younger infants, if not better. The observation that selective imitation precedes faithful imitation discounts the claim that children copy faithfully because they do not understand the causal structure of the task, and supports our conclusion that they do so to enhance and maintain the social interaction.

Our design also allowed us to address an alternative explanation for the observed relation between surgency and faithful imitation, namely that high-surgency infants may be more active in general, rather than being more faithful imitators specifically. If the relation between surgency and faithful imitation is due to higher activity levels of high-surgency infants, they should not only be copying more first actions (faithful imitations) they should also be more active with regard to copying second actions compared to their low-surgency counterparts. However, no effect of Surgency was found with regard to copying second actions, indicating that high-surgency infants do not just do more in general.

Future work could extend our current understanding of the differences between selective and faithful imitation in terms of their underlying motivations, the execution of faithful and selective imitation and the learning that arises from it. For instance, we know that both selective and faithful imitation draw on social understanding, as demonstrated by a variety of studies discussed in the introduction (e.g. Brugger et al., 2007; Carpenter et al., 2005; Gattis et al., 2002; Hoicka \& Gattis, 2008; Nielsen, 2006; Over \& Gattis, 2010). In addition, faithful imitation could also draw on social understanding with regard to children's increasing understanding of social norms and rules. Various studies with older children have demonstrated that children are capable of learning social norms and rules (Rakockzy, Warneken \& Tomasello, 2008; Rakockzy, Warneken \& Tomasello, 2009). It is possible that children who are more interested in interacting with other people (i.e. high-surgency/extraversion) are also more likely to follow the social norms and rules of other people and therefore copy more faithfully. Future work is needed to address whether surgency is related to following social norms and rules and whether infants as young as 12 and 15 months are capable of learning social norms and rules, because thus far 2-year-olds are the youngest age group for which research has found some indication of social norm learning.

Faithful imitation is sometimes referred to as overimitation, and defined specifically as copying causally unnecessary actions (Horner \& Whiten, 2005; Kenward, 2012; Kenward, Karlsson \& Persson, 2011; Lyons et al., 2007). While this empirical definition makes a valuable logical contribution, we propose that an exclusive focus on children's copying of unnecessary actions may be misleading. Defining faithful imitation as the imitation of causally unnecessary acts creates a puzzle about the supposedly illogical nature of children's behavior: 'But why would children do that?' Instead we proposed that faithful imitation is only meaningful when defined as the imitation of both causally necessary and causally unnecessary acts. The proposed correspondences between instrumental motivations and selective imitation and between social motivations and faithful imitation as defined by copying both necessary and unnecessary first actions, together with the results reported here, suggest that faithful imitation is instead a new way for a child to 
view the imitative encounter. As a result, faithful imitation is a new step in the social communicative life of a child.

The public sociologist Michael Burawoy has distinguished two types of knowledge (2005). Instrumental knowledge, according to Burawoy, is the product of identifying a problem and finding the solution. Reflexive knowledge, in contrast, involves taking a step back and reflecting on the aims of knowledge, and on the values of other people. Burawoy argues that producing instrumental knowledge can be a solitary activity, but reflexive knowledge involves social interactions because it requires a dialogue. Burawoy's contrast between instrumental and reflexive knowledge evokes Uzgiris' distinction between instrumental and social motivations. We want to draw on both distinctions, and suggest that imitation can arise from different motivations, and lead to different kinds of knowledge. Selective imitation involves identifying a problem and finding a solution. Selective imitation draws on causal knowledge as well as knowledge of goals and intentions to help find the problem solutions (Bekkering et al., 2000; Brugger et al., 2007; Carpenter et al., 1998, 2005; Gattis et al., 2002; Hoicka \& Gattis, 2008; Over \& Gattis, 2010). In contrast, faithful imitation is not primarily about solving a problem, but about reflecting on the knowledge and values of other people. Faithful imitation involves an awareness of the interpersonal nature of imitation, and as such, is a reflective and communicative act.

Interestingly, the observed shift towards faithful imitation begins around the age at which infants also begin to share goals and intentions with social partners. Tomasello et al. (2005) argued in their review that infants have a strong motivation to share emotional states from very early in life but that this motivation becomes more sophisticated as infants get older, with infants becoming able to share goals and perspectives with others from around 12 months onwards. The finding that the shift towards faithful imitation coincides with infants becoming able to share goals and perspectives with others strengthens the idea that infants imitate faithfully because of a motivation to create a shared experience with a social partner.

In this paper we have argued that the onset of faithful imitation is a new step in the social communicative life of a child. The present results demonstrate that infant imitation is influenced by causal structure from 12 months onwards as evidenced by selective imitation at both ages. Furthermore, the present results provide clear evidence that the onset of faithful imitation begins earlier than previously thought, namely between 12 and 15 months and that this faithful imitation is not due to a lack of causal understanding. Finally, our findings suggest a relation between faithful imitation and the social motivation to engage in and maintain social interactions with partners. Thus infants who are more like The Jungle Book's King Louie, with greater desire and willingness to engage in social interactions, are more faithful imitators. Future research should investigate this relation further and assess how these important developments in imitation at the beginning of the first year influence imitation at later ages.

\section{Acknowledgements}

This research was supported by the Leverhulme Trust, the Economic and Social Sciences Research Council and the ERC Advanced Grant \#269484 INTERACT. We thank Juan Carlos Gomez and three anonymous reviewers for their comments on previous versions of this manuscript, Elena Hoicka for her help with the analyses and Barbara Carotti for her help with coding of the data.

\section{References}

Barr, R., Dowden, A., \& Hayne, H. (1996). Developmental changes in deferred imitation by 6- to 24-month-old infants. Infant Behavior and Development, 19, 159-170.

Bates, D., Maechler, M., \& Dai, B. (2012). lme4: Linear mixedeffects models using S4 classes [Computer software manual]. Available from: http://lme4.r-forge.r-project.org/ (R package version 0.999999-0).

Bekkering, H., Wohlschläger, A., \& Gattis, M. (2000). Imitation of gestures in children is goal-directed. Quarterly Journal of Experimental Psychology, 53A, 153-164.

Brugger, A., Lariviere, L.A., Mumme, D.L., \& Bushnell, E.W. (2007). Doing the right thing: Infants' selection of actions to imitate from observed event sequences. Child Development, 78, 806-824.

Burawoy, M. (2005). 2004 American Sociological Association Presidential address: For public sociology. British Journal of Sociology, 56, 259-294.

Buss, A.H., \& Plomin, R. (1986). The EAS approach to temperament. In: R. Plomin \& J. Dunn (Eds.), The study of temperament: Changes, continuities and challenges (pp. 6779). Hillsdale, NJ: Lawrence Erlbaum Associates.

Calkins, D., Fox, N.A., \& Marshall, T.R. (1996). Behavioral and physiological antecedents of inhibited and uninhibited behaviour. Child Development, 67, 523-540.

Carpenter, M. (2006). Instrumental, social, and shared goals and intentions in imitation. In S.J. Rogers \& J. Williams (Eds.), Imitation and the development of the social mind: Lessons from typical development and autism (pp. 48-70). New York: Guilford Press.

Carpenter, M., Akhtar, N., \& Tomasello, M. (1998). Fourteenthrough 18-month-old infants differentially imitate inten- 
tional and accidental actions. Infant Behavior and Development, 21, 315-330.

Carpenter, M., Call, J., \& Tomasello, M. (2005). Twelve- and 18 -month-olds copy actions in terms of goals. Developmental Science, 8, 13-20.

Chartrand, T.L., \& Bargh, J.A. (1999). The chameleon effect: the perception-behavior link and social interaction. Journal of Personality and Social Psychology, 76, 893-910.

Cheek, J.M., \& Buss, A.H. (1981). Shyness and sociability. Journal of Personality and Social Psychology, 41, 330-339.

Ellis-Davies, K., Sakkalou, E., Fowler, N.C., Hilbrink, E.E., \& Gattis, M. (2012). CUE: the continuous unified electronic diary method. Behavior Research Methods, 44, 1063-1078.

Gardiner, A.K., Greif, M.L., \& Bjorklund, D.F. (2011). Guided by intention: preschoolers' imitation reflects inferences of causation. Journal of Cognition and Development, 12, 355-373.

Gartstein, M.A., \& Rothbart, M.K. (2003). Studying infant temperament via the Revised Infant Behaviour Questionnaire. Infant Behavior and Development, 26, 64-86.

Gattis, M., Bekkering, H., \& Wohlschläger, A. (2002). Goaldirected imitation. In A. Meltzoff \& W. Prinz (Eds.), The imitative mind (pp. 183-205). Cambridge: Cambridge University Press.

Goldsmith, H.H., Buss, A.H., Plomin, R., Rothbart, M.K., Thomas, A., Chess, S., Hinde, R.A., \& McCall, R.B. (1987). Roundtable: What is temperament? Four approaches Child Development, 58, 505-529.

Grist, L.C., \& McCord, D.M. (2010). Individual differences in preschool children: temperament or personality. Infant and Child Development, 19, 264-274.

Hoicka, E.K., \& Akhtar, N. (2011). Preschoolers joke with jokers, but correct foreigners. Developmental Science, 14, 848-858.

Hoicka, E.K., \& Gattis, M. (2008). Do the wrong thing: how toddlers tell a joke from a mistake. Cognitive Development, 23, 180-190.

Horner, V., \& Whiten, A. (2005). Causal knowledge and imitation/emulation switching in chimpanzees (Pan troglodytes) and children (Homo sapiens). Animal Cognition, 8, 164-181.

Jaeger, T.F. (2008). Categorical data analysis: away from ANOVAs (transformation or not) and toward logit mixed models. Journal of Memory and Language, 59, 434-446.

Kenward, B. (2012). Over-imitating preschoolers believe unnecessary actions are normative and enforce their performance by a third party. Journal of Experimental Child Psychology, 112, 195-207.

Kenward, B., Karlsson, M., \& Persson, J. (2011). Overimitation is better explained by norm learning than by distorted causal learning. Proceedings of the Royal Society B: Biological Sciences, 278, 1239-1246.

Killen, M., \& Uzgiris, I.C. (1981). Imitations of actions with objects: the role of social meaning. Journal of Genetic Psychology, 138, 219-229.

Kuhl, P.K., \& Meltzoff, A.N. (1996). Infant vocalizations in response to speech: vocal imitation and developmental change. Journal of Acoustical Society of America, 100, 2425-2438.

Lyons, D.E., Damrosch, D.H., Lin, J.K., Macris, D.M., \& Keil, F.C. (2011). The scope and limits of overimitation in the transmission of artefact culture. Philosophical Transactions of the Royal Society, B, 366, 1158-1167.

Lyons, D.E., Young, A.G., \& Keil, F.C. (2007). The hidden structure of overimitation. Proceedings of the National Academy of Sciences, USA, 104 (50), 19751-19756.

Meltzoff, A.N., \& Moore, M.K. (1983). Newborn infants imitate adult facial gestures. Child Development, 54, 702-709.

Nielsen, M. (2006). Copying actions and copying outcomes: social learning through the second year. Developmental Psychology, 42, 555-565.

Nielsen, M. (2009). The imitative behavior of children and chimpanzees: a window on the transmission of cultural traditions, Revue de primatologie, 1, 2-13.

Nielsen, M., \& Blank, C. (2011). Imitation in young children: when who gets copied is more important than what gets copied. Developmental Psychology, 47, 1050-1053.

Nielsen, M., Simcock, G., \& Jenkins, L. (2008). The effect of social engagement on 24-month-olds' imitation from live and televised models. Developmental Science, 11, 722-731.

Over, H., \& Carpenter, M. (2009). Priming third-party ostracism increases affiliative imitation in children. Developmental Science, 12, F1-F8.

Over, H., \& Carpenter, M. (2012). Putting the social into social learning: explaining both selectivity and fidelity in children's copying behavior. Journal of Comparative Psychology, 126, 182-192.

Over, H., \& Gattis, M. (2010). Verbal imitation is based on intention understanding. Cognitive Development, 25, 46-55.

Perra, O., \& Gattis, M. (2008). Reducing the mapping between perception and action facilitates imitation. British Journal of Developmental Psychology, 26, 133-144.

Putnam, S.P., Gartstein, M.A., \& Rothbart, M.K. (2006). Measurement of fine-grained aspects of toddler temperament: the early childhood behavior questionnaire. Infant Behavior and Development, 29, 386-401.

Putnam, S.P., Rothbart, M.K., \& Gartstein, M.A. (2008). Homotypic and heterotypic continuity of fine-grained temperament during infancy, toddlerhood, and early childhood. Infant and Child Development, 17, 387-405.

Rakoczy, H., Warneken, F., \& Tomasello, M. (2008). Theo sources of normativity: young children's awareness of the normative structure of games. Developmental Psychology, 44, 875-881.

Rakoczy, H., Warneken, F., \& Tomasello, M. (2009). Young children's selective learning of rule games from reliable and unreliable models. Cognitive Development, 24, 61-69.

R Development Core Team (2012). R: a language and environment for statistical computing. R Foundation for Statistical Computing, Vienna, Austria. URL http://www.R-project.org/

Rothbart, M.K., Ahadi, S.A., Hershey, K.L., \& Fisher, P. (2001). Investigations of temperament at three to seven years: the Children's Behavior Questionnaire. Child Development, 72, 1394-1408. 
Sakkalou, E., Ellis-Davies, K., Fowler, N.C., Hilbrink, E.E., \& Gattis, M. (2013). Infants show stability of goal-directed imitation. Journal of Experimental Child Psychology, 114, 1-9.

Sakkalou, E., \& Gattis, M. (2012). Infants infer intentions from prosody. Cognitive Development, 27, 1-16.

Schmidt, L.A., \& Fox, N.A. (1994). Patterns of cortical electrophysiology and autonomic activity in adults' shyness and sociability. Biological Psychology, 38, 183-198.

Tomasello, M., Carpenter, M., Call, J., Behne, T., \& Moll, H. (2005). Understanding and sharing of intentions: the origins of cultural cognition. Behavioral and Brain Sciences, 28, 675-735.
Uzgiris, I.C. (1981). Two functions of imitation during infancy. International Journal of Behavioral Development, 4, 1-12.

Want, S.C., \& Harris, P.L. (2002). How do children ape? Applying concepts from the study of non-human primates to the developmental study of 'imitation' in children. Developmental Science, 5, 1-41.

Received: 14 February 2011

Accepted: 23 February 2013 